EUROPEAN JOURNAL OF PURE AND APPLIED MATHEMATICS

Vol. 12, No. 1, 2019, 58-78

ISSN 1307-5543 - www.ejpam.com

Published by New York Business Global

\title{
Backwards Itô-Henstock Integral for the Hilbert-Schmidt-Valued Stochastic Process
}

\author{
Ricky F. Rulete ${ }^{1}$, Mhelmar A. Labendia ${ }^{2, *}$ \\ 1 Department of Mathematics and Statistics, College of Arts and Sciences, \\ University of Southeastern Philippines, Bo. Obrero, 8000 Davao City, Philippines \\ 2 Department of Mathematics and Statistics, College of Science and Mathematics, \\ Mindanao State University-Iligan Institute of Technology, 9200 Iligan City, Philippines
}

\begin{abstract}
In this paper, a definition of backwards Itô-Henstock integral for the Hilbert-Schmidtvalued stochastic process is introduced. We formulate the Itô isometry for this integral. Moreover, an equivalent definition for this integral is given using the concept of $A C^{2}[0, T]$-property, a version of absolute continuity.
\end{abstract}

2010 Mathematics Subject Classifications: 60H30, 60H05

Key Words and Phrases: Backwards Itô-Henstock integral, Itô Isometry, $A C^{2}$-property

\section{Introduction}

The most well-known integral is the Riemann integral. It was formulated by Bernhard Riemann in 1850. This is the first integral introduced to most students in the study of elementary calculus. However, the class of Riemann-integrable functions is quite limited. Henri Lebesgue attempts to solve some of the shortcomings of the Riemann integral. However, for non-mathematicians the Lebesgue integral is difficult to understand and requires enough background of measure theory. In 1950s, a Riemann-type integral was discovered independently by R. Henstock and J. Kurzwiel. This integral includes Riemann and that of Lebesgue. This integral is now known as Henstock-Kurzwiel or HK integral. In this paper, however, we will call this integral simply as Henstock integral. The Henstock integral used non-uniform meshes in contrast to Riemann. Such technique turns out to encompass the classical stochastic integral, see([7], [8], [9], [13] and [14]]). This technique is now known as the Henstock approach.

In stochastic calculus, the stochastic integral of a real-valued adapted process is obtained from the mean square limit of stochastic integrals of simple processes, see [16].

${ }^{*}$ Corresponding author.

DOI: https://doi.org/10.29020/nybg.ejpam.v12i1.3342

Email addresses: ricky_rulete@yahoo.com.ph (R. Rulete), mhelmar.labendia@g.msuiit.edu.ph (M. Labendia) 
This is the classical approach to stochastic integration which is almost similar in defining the Lebesgue integral of a measurable function. Hence, Henstock approach to stochastic integration have been studied in several papers see([15], [17], [21], [22] and [23]) since it gives more explicit definition, reduces the technicalities in the classical way of defining the stochastic integral and is less measure theoretic.

In [6], [19], and [18], the concept of stochastic integral has been extended to infinitedimensional spaces, namely Hilbert and Banach spaces. In a Hilbert space, the stochastic integral is presented in a manner similar to the real-valued case. The integrator is $Q$ Wiener process, a Hilbert space-valued Wiener process which is dependent on a symmetric nonnegative trace-class operator $Q$ and the integrand is an operator-valued stochastic process. In a general Banach space, however, there seems to be no unifying treatment of stochastic integration.

In 2018, Labendia, et.al. [11], introduced the (forward) Itô-Henstock integral of an operator-valued stochastic process with respect to a Hilbert space-valued $Q$-Wiener process. This integral uses (forward) filtration. Moreover, the $\delta$-fine partial division is belated in the sense that the associated points (or tags) are always on the left endpoints of the subintervals. They formulated a version of Itô's formula and gave an alternative definition of the classical Itô integral of an $L(U, V)$-valued stochastic process using Henstock approach, where $U$ and $V$ are separable Hilbert spaces and $L(U, V)$ is the space of all bounded linear operators $Q: U \rightarrow V$. In [10], the (forward) Itô-Henstock integral has been characterized using $A C^{2}[0, T]$-property, a version of absolute continuity.

The backwards Itô integral with respect to a Brownian motion was defined by Arcede and Cabral in 2011, see [3]. In this integral, all processes start at a fix time $T>0$ and then proceed backwards to some earlier time $s$. Henstock approach was used together with the notions of backwards $\delta$-fine partial division (backwards in the sense that the tags are the right endpoints of the disjoint left-open subintervals) and backwards filtration. One of their results are the fundamental theorem of calculus, integration-by-parts and the Itô formula for backwards Itô integral see([4], [5]).

In this paper, we define the backwards Itô-Henstock integral of an operator-valued stochastic process with respect to a Hilbert space-valued $Q$-Wiener process which is actually an extension of the work of Arcede and Cabral in [3]. Here, we formulate the Itô isometry and give an equivalent definition using the concept of $A C^{2}$ property, a version of absolute continuity.

\section{Preliminaries}

Throughout this paper, $\mathbb{R}$ denotes the set of real numbers, $\mathbb{R}_{0}^{+}$denotes the set of nonnegative real numbers, $\mathbb{N}$ the set of positive integers and $\{\Omega, \mathcal{G}, \mathbb{P}\}$ denotes a probability space.

Let $\left\{\mathcal{G}_{t}: 0 \leq t \leq T\right\}$ be a family of sub $\sigma$-field of $\mathcal{G}$. Then $\left\{\mathcal{G}_{t}: 0 \leq t \leq T\right\}$ is called a backwards filtration if $\mathcal{G}_{t} \subseteq \mathcal{G}_{s}$ for all $0 \leq s \leq t \leq T$. If in addition, $\left\{\mathcal{G}_{t}: 0 \leq t \leq T\right\}$ satisfies the following condition: (1) $\mathcal{G}_{T}$ contains all sets of $\mathbb{P}$-measure zero in $\mathcal{G}$; and $(2)$ for each $t \in[0, T], \mathcal{G}_{t}=\mathcal{G}_{t-}:=\bigcap_{s<t} \mathcal{G}_{s}$. Then $\left\{\mathcal{G}_{t}: 0 \leq t \leq T\right\}$ is called a standard 
backwards filtration. We often write $\left\{\mathcal{G}_{t}\right\}$ instead of $\left\{\mathcal{G}_{t}: 0 \leq t \leq T\right\}$. See [1].

Let $H$ be a separable Banach space. A stochastic process $f$ or simply process is a function $f:[0, T] \times \Omega \rightarrow H$, where $[0, T]$ is an interval in $\mathbb{R}_{0}^{+}$and $f(\cdot, t)$ is $\mathcal{G}_{t}$-measurable for each $t \in[0, T]$. A process $f=\left\{f_{t}: t \in[0, T]\right\}$ is said to be backwards adapted to a standard backwards filtration $\left\{\mathcal{G}_{t}\right\}$ if $f_{t}$ is $\mathcal{G}_{t}$-measurable for each $t \in[0, T]$.

Let $U$ and $V$ be separable Hilbert spaces. Denote $L(U, V)$ the space of all bounded linear operators from $U$ to $V, L(U):=L(U, U), Q u:=Q(u)$ if $Q \in L(U, V)$, and $L^{2}(\Omega, V)$ the space of all square-integrable random variables from $\Omega$ to $V$. An operator $Q \in L(U)$ is said to be self-adjoint or symmetric if for all $u, u^{\prime} \in U,\left\langle Q u, u^{\prime}\right\rangle_{U}=\left\langle u, Q u^{\prime}\right\rangle_{U}$ and is said to be nonnegative definite if for every $u \in U,\langle Q u, u\rangle_{U} \geq 0$.

Let $\left\{e_{j}\right\}_{j=1}^{\infty}$, or simply $\left\{e_{j}\right\}$, be an orthonormal basis (abbrev. as ONB) in $U$. If $Q \in L(U)$ is nonnegative definite, then the trace of $Q$ is defined by $\operatorname{tr} Q=\sum_{j=1}^{\infty}\left\langle Q e_{j}, e_{j}\right\rangle_{U}$. It is shown in [20] that $\operatorname{tr} Q$ is well-defined and may be defined in terms of an arbitrary ONB. Moreover, there exists a unique operator $Q^{\frac{1}{2}} \in L(U)$ such that $Q^{\frac{1}{2}}$ is nonnegative definite and $\left(Q^{\frac{1}{2}}\right)^{2}=Q$. An operator $Q: U \rightarrow U$ is said to be trace-class if $\operatorname{tr}[Q]:=$ $\operatorname{tr}\left(Q Q^{*}\right)^{\frac{1}{2}}<\infty$. If $Q \in L(U)$ is a symmetric nonnegative definite trace-class operator, then there exists an ONB $\left\{e_{j}\right\} \subset U$ and a sequence of nonnegative real numbers $\left\{\lambda_{j}\right\}$ such that $Q e_{j}=\lambda_{j} e_{j}$ for all $j \in \mathbb{N}$, and $\lambda_{j} \rightarrow 0$ as $j \rightarrow \infty$ [20, p.203]. We shall call the sequence of pairs $\left\{\lambda_{j}, e_{j}\right\}$ an eigensequence defined by $Q$.

Let $Q: U \rightarrow U$ be a symmetric nonnegative definite trace-class operator and let $\left\{\lambda_{j}, e_{j}\right\}$ be an eigensequence defined by $Q$. Then the subspace $U_{Q}:=Q^{\frac{1}{2}} U$ of $U$ equipped with the inner product $\langle u, v\rangle_{U_{Q}}=\left\langle Q^{-1 / 2} u, Q^{-1 / 2} v\right\rangle_{U}$, where $Q^{1 / 2}$ is being restricted to $\left[\operatorname{Ker} Q^{1 / 2}\right]^{\perp}$ is a separable Hilbert space with $\left\{\sqrt{\lambda_{j}} e_{j}\right\}$ as its ONB, see [18, p.90], [6, p.23]. Let $\left\{f_{j}\right\}$ be an ONB in $U_{Q}$. An operator $S \in L\left(U_{Q}, V\right)$ is said to be Hilbert-Schmidt if $\sum_{j=1}^{\infty}\left\|S f_{j}\right\|_{V}^{2}=\sum_{j=1}^{\infty}\left\langle S f_{j}, S f_{j}\right\rangle_{V}<\infty$. Denote by $L_{2}\left(U_{Q}, V\right)$ the space of all HilbertSchmidt operators from $U_{Q}$ to $V$, which is known [19, p.112] to be a separable Hilbert space with norm $\|S\|_{L_{2}\left(U_{Q}, V\right)}=\sqrt{\sum_{j=1}^{\infty}\left\|S f_{j}\right\|_{V}^{2}}$. The Hilbert-Schmidt operator $S \in L_{2}\left(U_{Q}, V\right)$ and the norm $\|S\|_{L_{2}\left(U_{Q}, V\right)}$ may be defined in terms of an arbitrary ONB, see [18, p.418], [19, p.111]. It is shown in [6, p.25] that $L(U, V)$ is properly contained in $L_{2}\left(U_{Q}, V\right)$.

We fix an element $Q \in L(U)$, symmetric nonnegative definite trace-class operator. A $U$-valued stochastic process $W_{t}, t \in[0, T]$, on a probability space $(\Omega, \mathcal{G}, \mathbb{P})$ is called a $Q$-Wiener process in $U$ if:

(i) $W(0, \omega)=0_{U}$ for each $\omega \in \Omega$,

(ii) $W$ has $\mathbb{P}$-almost surely (abbrev. as $\mathbb{P}$-a.s.) continuous trajectories, i.e.,

$$
W(\cdot, \omega):[0, T] \rightarrow U \text { is } \mathbb{P} \text {-a.s. continuous }
$$

(iii) the increments of $W$ are independent, i.e. the random variables

$$
W_{t_{1}}, W_{t_{2}}-W_{t_{1}}, W_{t_{3}}-W_{t_{2}}, \ldots, W_{t_{n}}-W_{t_{n-1}}
$$

are independent for all $0 \leq t_{1}<\cdots<t_{n} \leq T, n \in N$, 
(iv) the increments have the following Gaussian laws:

$$
\mathbb{P} \circ\left(W_{t}-W_{s}\right)^{-1}=\mathcal{N}(0,(t-s) Q) \text { for all } 0 \leq s \leq t \leq T .
$$

By Proposition 4.2 (see $[18$, p.88]), such a $Q$-Wiener process exists.

We define $\mathcal{N}:=\{A \in \mathcal{G} \mid \mathbb{P}(A)=0\}, \tilde{\mathcal{G}}_{t}:=\sigma\left(W_{T}-W_{s} \mid t \leq s \leq T\right), \tilde{\mathcal{G}}_{t}^{0}:=\sigma\left(\tilde{\mathcal{G}}_{t} \cup \mathcal{N}\right)$ and

$$
\mathcal{G}_{t}:=\bigcap_{s<t} \tilde{\mathcal{G}}_{s}^{0}, \quad t \in[0, T] .
$$

Since $\mathcal{N} \subseteq \tilde{\mathcal{G}}_{s}^{0}$ for all $s \in[0, T]$ and $\left\{\mathcal{G}_{t}\right\}_{0 \leq t \leq T}$ is decreasing, we have the following result:

Proposition 1. Let $t \in[0, T]$. Then the filtration $\mathcal{G}_{t}$ given in (1) is a standard backwards filtration.

We note that the distance from an element $u \in U$ to a nonempty subset $A \subset U$, denoted by $\operatorname{dist}(u, A)$ is defined to be

$$
\operatorname{dist}(u, A)=\inf _{a \in A}\|u-a\|_{U} .
$$

Proposition 2. Let $W_{t}, t \in[0, T]$, be an arbitrary $U$-valued $Q$-Wiener process on a probability space $(\Omega, \mathcal{G}, \mathbb{P})$. Then $W_{t}-W_{s}$ is independent of $\mathcal{G}_{t}$ for all $0 \leq s \leq t \leq T$, where $\mathcal{G}_{t}$ is given in $(1)$.

Proof. Let $0 \leq s \leq t \leq T$. Since a $U$-valued $Q$-Wiener process has independent increments, $W_{t}-W_{s}$ and $W_{T}-W_{t}$ are independent. It follows that $W_{t}-W_{s}$ and $W_{T}-W_{t}^{\prime}$ are independent for all $t \leq t^{\prime} \leq T$. Hence, $W_{t}-W_{s}$ is independent of $\sigma\left(W_{T}-W_{t}^{\prime}: t \leq t^{\prime} \leq\right.$ $T)=\tilde{\mathcal{G}}_{t}$. Also, $W_{t}-W_{s}$ is independent of $\tilde{\mathcal{G}}_{t}^{0}$. To prove now that $W_{t}-W_{s}$ is independent of $\mathcal{G}_{t}$ it is enough to show that

$$
\mathbb{P}\left(\left\{W_{t}-W_{s} \in A\right\} \cap B\right)=\mathbb{P}\left(\left\{W_{t}-W_{s} \in A\right\}\right) \cdot \mathbb{P}(B)
$$

for any $B \in \mathcal{G}_{t}$ and any closed subset $A \in U$ as $\{A \subset U \mid A$ closed $\}$ generates $\mathcal{B}(U)$ and is stable under finite intersection. But we have

$$
\mathbb{P}\left(\left\{W_{t}-W_{s} \in A\right\} \cap B\right)=\mathbb{E}\left[1_{\left\{W_{t}-W_{s} \in A\right\}} \cdot 1_{B}\right]=\mathbb{E}\left[1_{A} \circ\left(W_{t}-W_{s}\right) \cdot 1_{B}\right] .
$$

Let $F: \Omega \rightarrow\{0,1\}$ be defined by

$$
F(\omega)=\lim _{n \rightarrow \infty}\left(1-n \operatorname{dist}\left(\left(W_{t}-W_{s}\right)(\omega), A\right)\right) \vee 0, \quad \omega \in \Omega
$$

where $\vee$ denotes "maximum". Let $\omega \in \Omega$. If $\left(W_{t}-W_{s}\right)(\omega) \in A$, then $\operatorname{dist}\left(\left(W_{t}-\right.\right.$ $\left.\left.W_{s}\right)(\omega), A\right)=0$ and $F(\omega)=1=\left(1_{A} \circ W_{t}-W_{s}\right)(\omega)$. Otherwise, $\operatorname{dist}\left(\left(W_{t}-W_{s}\right)(\omega), A\right)>0$ and $F(\omega)=0=\left(1_{A} \circ W_{t}-W_{s}\right)(\omega)$. Hence, $1_{A} \circ\left(W_{t}-W_{s}\right)=F$. So, we have

$$
\mathbb{P}\left(\left\{W_{t}-W_{s} \in A\right\} \cap B\right)=\mathbb{E}\left[\left(\lim _{n \rightarrow \infty}\left(1-n \operatorname{dist}\left(W_{t}-W_{s}, A\right)\right) \vee 0\right) \cdot 1_{B}\right]
$$




$$
\begin{aligned}
& =\mathbb{E}\left[\lim _{n \rightarrow \infty}\left(\left(1-n \operatorname{dist}\left(W_{t}-W_{s}, A\right)\right) \vee 0\right) \cdot 1_{B}\right] \\
& =\lim _{n \rightarrow \infty} \mathbb{E}\left[\left(\left(1-n \operatorname{dist}\left(W_{t}-W_{s}, A\right)\right) \vee 0\right) \cdot 1_{B}\right] .
\end{aligned}
$$

Moreover, for each $\omega \in \Omega$, we have

$$
\begin{aligned}
\operatorname{dist}\left(\left(W_{t}-W_{s}\right)(\omega), A\right) & =\inf _{a \in A}\left\|\left(W_{t}-W_{s}\right)(\omega)-a\right\|_{U} \\
& =\inf _{a \in A}\left\|\lim _{m \rightarrow \infty} W_{t-\frac{1}{m}}(\omega)-W_{s}(\omega)-a\right\|_{U} \\
& =\lim _{m \rightarrow \infty} \inf _{a \in A}\left\|W_{t-\frac{1}{m}}(\omega)-W_{s}(\omega)-a\right\|_{U} \\
& =\lim _{m \rightarrow \infty} \operatorname{dist}\left(\left(W_{t-\frac{1}{m}}-W_{s}\right)(\omega), A\right) .
\end{aligned}
$$

This implies that

$$
\begin{aligned}
\mathbb{P}\left(\left\{W_{t}-W_{s} \in A\right\} \cap B\right) & =\lim _{n \rightarrow \infty} \mathbb{E}\left[\left(\left(1-n \lim _{m \rightarrow \infty} \operatorname{dist}\left(W_{t-\frac{1}{m}}-W_{s}, A\right)\right) \vee 0\right) \cdot 1_{B}\right] \\
& =\lim _{n \rightarrow \infty} \mathbb{E}\left[\lim _{m \rightarrow \infty}\left(\left(1-n \operatorname{dist}\left(W_{t-\frac{1}{m}}-W_{s}, A\right)\right) \vee 0\right) \cdot 1_{B}\right] \\
& =\lim _{n \rightarrow \infty} \lim _{m \rightarrow \infty} \mathbb{E}\left[\left(\left(1-n \operatorname{dist}\left(W_{t-\frac{1}{m}}-W_{s}, A\right)\right) \vee 0\right) \cdot 1_{B}\right] .
\end{aligned}
$$

Since $W_{t-\frac{1}{m}}-W_{s}$ is independent of $\tilde{\mathcal{G}}_{t-\frac{1}{m}}^{0} \supseteq \mathcal{G}_{t}$ if $m$ is large, we have

$$
\begin{aligned}
\mathbb{P}\left(\left\{W_{t}-W_{s} \in A\right\} \cap B\right) & =\lim _{n \rightarrow \infty} \lim _{m \rightarrow \infty} \mathbb{E}\left[\left(1-n \operatorname{dist}\left(W_{t-\frac{1}{m}}-W_{s}, A\right)\right) \vee 0\right] \cdot \mathbb{E}\left[1_{B}\right] \\
& =\mathbb{P}\left(\left\{W_{t}-W_{s} \in A\right\}\right) \cdot \mathbb{P}(B) .
\end{aligned}
$$

This completes the proof.

From now onwards, the backwards filtered probability shall mean a filtered probability space such that $W_{t}$ is adapted to $\mathcal{G}_{t}$ and $W_{t}-W_{s}$ is independent of $\mathcal{G}_{t}$ for all $0 \leq s \leq t \leq T$.

\section{Backwards Itô-Henstock Integral}

In this section, we shall present the backwards Itô-Henstock integral and some related results.

Let $\delta$ be a positive function on $(0, T]$. A finite collection $D=\left\{\left(\left(u_{i}, \xi_{i}\right], \xi_{i}\right)\right\}_{i=1}^{n}$ of interval-point pairs is said to be a backwards partial division of $[0, T]$ if $\left\{\left(u_{i}, \xi_{i}\right]\right\}_{i=1}^{n}$ is a finite collection of disjoint subintervals of $(0, T]$. An interval-point pair $((u, \xi], \xi)$ is said to be backwards $\delta$-fine if $(u, \xi] \subseteq(\xi-\delta(\xi), \xi]$, whenever $(u, \xi] \subseteq(0, T]$ and $\xi \in(0, T]$. We call $D=\left\{\left(\left(u_{i}, \xi_{i}\right], \xi_{i}\right)\right\}_{i=1}^{n}$ a backwards $\delta$-fine partial division of $[0, T]$ if $D$ is a backwards partial division of $[0, T]$ and for each $i$, the interval-point pair $\left(\left(u_{i}, \xi_{i}\right], \xi_{i}\right)$ is backwards $\delta$-fine. 
We note that given any positive function $\delta$, one may not be able to find a full division that covers the entire interval $(0, T]$. For instance, let $\delta(\xi)=\xi / 2$. Then the interval $(0, T]$ cannot be covered by any finite collection of backwards $\delta$-fine intervals.

Given $\eta>0$, a given backwards $\delta$-fine partial division $D=\left\{\left(\left(u_{i}, \xi_{i}\right], \xi_{i}\right)\right\}_{i=1}^{n}$ is said to be backwards $(\delta, \eta)$-fine partial division of $[0, T]$ if it fails to cover $(0, T]$ by at most length $\eta$, that is,

$$
\left|T-(D) \sum_{i=1}^{n}\left(\xi_{i}-u_{i}\right)\right| \leq \eta
$$

We are now ready to define the backwards Itô-Henstock integral. Throughout the following discussions, assume that $U$ and $V$ are separable Hilbert spaces, $Q: U \rightarrow U$ is a symmetric nonnegative definite trace-class operator, $\left\{\lambda_{j}, e_{j}\right\}$ is an eigensequence defined by $Q$, and $W$ is a $U$-valued $Q$-Weiner process.

Definition 1. Let $f:[0, T] \times \Omega \rightarrow L_{2}\left(U_{Q}, V\right)$ be a backwards adapted process. Then $f$ is said to be backwards Itô-Henstock integrable, or $\mathcal{I H}_{B}$-integrable, on $[0, T]$ with respect to $W$ if there exists $A \in L^{2}(\Omega, V)$ such that for every $\varepsilon>0$, there is a positive function $\delta$ on $(0, T]$ and a positive number $\eta$ such that for any backwards $(\delta, \eta)$-fine partial division $D=\left\{\left(\left(u_{i}, \xi_{i}\right], \xi_{i}\right)\right\}_{i=1}^{n}$ of $[0, T]$, we have

$$
\mathbb{E}\left[\|S(f, D, \delta, \eta)-A\|_{V}^{2}\right]<\varepsilon
$$

where

$$
S(f, D, \delta, \eta):=(D) \sum f_{\xi}\left(W_{\xi}-W_{u}\right):=\sum_{i=1}^{n} f_{\xi_{i}}\left(W_{\xi_{i}}-W_{u_{i}}\right) .
$$

In this case, $f$ is $\mathcal{I H}_{B}$-integrable to $A$ on $[0, T]$ and $A$ is called the $\mathcal{I H}_{B}$-integral of $f$ which will be denoted by $\left(\mathcal{I H}_{B}\right) \int_{0}^{T} f_{t} d W_{t}$ or $\left(\mathcal{I H}_{B}\right) \int_{0}^{T} f d W$.

Refer to [11, Lemma 3.5 and Lemma 3.6] for the proofs of the following two lemmas. When we speak of a subinterval of $[0, T]$, we shall mean that the subinterval is either a closed interval $[v, \xi]$ or half-open interval $(v, \xi]$.

Lemma 1. Let $f:[0, T] \times \Omega \rightarrow L_{2}\left(U_{Q}, V\right)$ be a backwards adapted process and $\left\{\left[v_{i}, \xi_{i}\right]\right\}_{i=1}^{n}$ be a finite collection of disjoint subintervals of $[0, T]$. Then

$$
\mathbb{E}\left[\sum_{i<p}\left\langle f_{\xi_{i}}\left(W_{\xi_{i}}-W_{v_{i}},\right), f_{\xi_{p}}\left(W_{\xi_{p}}-W_{v_{p}}\right)\right\rangle_{V}\right]=0 .
$$

Lemma 2. Let $f:[0, T] \times \Omega \rightarrow L_{2}\left(U_{Q}, V\right)$ be a backwards adapted process and $\left\{\left[v_{i}, \xi_{i}\right]\right\}_{i=1}^{n}$ be a finite collection of disjoint subintervals of $[0, T]$. Then

$\mathbb{E}\left[\left\|\sum_{i=1}^{n} f_{\xi_{i}}\left(W_{\xi_{i}}-W_{v_{i}}\right)\right\|_{V}^{2}\right]=\sum_{i=1}^{n} \mathbb{E}\left[\left\|f_{\xi_{i}}\left(W_{\xi_{i}}-W_{v_{i}}\right)\right\|_{V}^{2}\right]=\sum_{i=1}^{n}\left(\xi_{i}-v_{i}\right) \mathbb{E}\left[\left\|f_{\xi_{i}}\right\|_{L_{2}\left(U_{Q}, V\right)}^{2}\right]$. 
Theorem 1. Let $v, \xi \in[0, T]$ with $v<\xi$. Then

(i) $\mathbb{E}\left[\left\|W_{\xi}-W_{v}\right\|_{U}^{2}\right]=(\xi-v) \operatorname{tr} Q ;$

(ii) $\mathbb{E}\left[\left\|W_{\xi}-W_{v}\right\|_{U}^{4}\right]=(\xi-v)^{2}\left(2 \sum_{j=1}^{\infty} \lambda_{j}^{2}+(\operatorname{tr} Q)^{2}\right)$.

Proof. Let $v, \xi \in[0, T]$ with $v<\xi$. Then

(i) $\mathbb{E}\left[\left\|W_{\xi}-W_{v}\right\|_{U}^{2}\right]=\mathbb{E}\left[\sum_{j=1}^{\infty}\left\langle W_{\xi}-W_{v}, e_{j}\right\rangle_{U}^{2}\right]=\sum_{j=1}^{\infty} \mathbb{E}\left[\left\langle W_{\xi}-W_{v}, e_{j}\right\rangle_{U}^{2}\right]$. Since $\frac{\left\langle W_{t}, e_{j}\right\rangle_{U}}{\sqrt{\lambda_{j}}}$ is a Brownian motion,

$$
\mathbb{E}\left[\left(\frac{\left\langle W_{\xi}-W_{v}, e_{j}\right\rangle_{U}}{\sqrt{\lambda_{j}}}\right)^{2}\right]=\xi-v
$$

Hence,

$$
\mathbb{E}\left[\left\|W_{\xi}-W_{v}\right\|_{U}^{2}\right]=\sum_{j=1}^{\infty}(\xi-v) \lambda_{j}=(\xi-v) \sum_{j=1}^{\infty} \lambda_{j}=(\xi-v) \operatorname{tr} Q
$$

(ii) Note that

$$
\begin{aligned}
\mathbb{E}\left[\left\|W_{\xi}-W_{v}\right\|_{U}^{4}\right] \\
=\mathbb{E}\left[\left(\sum_{j=1}^{\infty}\left\langle W_{\xi}-W_{v}, e_{j}\right\rangle_{U}^{2}\right)^{2}\right] \\
=\mathbb{E}\left[\sum_{j=1}^{\infty}\left\langle W_{\xi}-W_{v}, e_{j}\right\rangle_{U}^{4}+\sum_{j \neq l}\left\langle W_{\xi}-W_{v}, e_{j}\right\rangle_{U}^{2}\left\langle W_{\xi}-W_{v}, e_{l}\right\rangle_{U}^{2}\right] \\
=\sum_{j=1}^{\infty} \mathbb{E}\left\langle W_{\xi}-W_{v}, e_{j}\right\rangle_{U}^{4}+\sum_{j \neq l} \mathbb{E}\left[\left\langle W_{\xi}-W_{v}, e_{j}\right\rangle_{U}^{2}\right] \mathbb{E}\left[\left\langle W_{\xi}-W_{v}, e_{l}\right\rangle_{U}^{2}\right] .
\end{aligned}
$$

Since $\frac{\left\langle W_{t}, e_{j}\right\rangle_{U}}{\sqrt{\lambda_{j}}}$ is a Brownian motion,

$$
\mathbb{E}\left[\left(\frac{\left\langle W_{\xi}-W_{v}, e_{j}\right\rangle_{U}}{\sqrt{\lambda_{j}}}\right)^{4}\right]=3(\xi-v)^{2} \quad \text { and } \quad \mathbb{E}\left[\left(\frac{\left\langle W_{\xi}-W_{v}, e_{j}\right\rangle_{U}}{\sqrt{\lambda_{j}}}\right)^{2}\right]=\xi-v \text {. }
$$


Thus,

$$
\begin{aligned}
\mathbb{E}\left[\left\|W_{\xi}-W_{v}\right\|_{U}^{4}\right] & =\sum_{j=1}^{\infty} 3 \lambda_{j}^{2}(\xi-v)^{2}+\sum_{j \neq l} \lambda_{j} \lambda_{l}(\xi-v)^{2} \\
& =(\xi-v)^{2}\left[2 \sum_{j=1}^{\infty} \lambda_{j}^{2}+\left(\sum_{j=1}^{\infty} \lambda_{j}^{2}+\sum_{j \neq l} \lambda_{j} \lambda_{l}\right)\right] \\
& =(\xi-v)^{2}\left[2 \sum_{j=1}^{\infty} \lambda_{j}^{2}+\left(\sum_{j=1}^{\infty} \lambda_{j}\right)^{2}\right] \\
& =(\xi-v)^{2}\left[2 \sum_{j=1}^{\infty} \lambda_{j}^{2}+(\operatorname{tr} Q)^{2}\right]
\end{aligned}
$$

Thereby, completing the proof.

Example 1. Let $W:[0, T] \times \Omega \rightarrow U$ be a $Q$-Weiner process. Then $\left\langle W_{t}, \cdot\right\rangle_{U}$ is $\mathcal{I H}_{B^{-}}$ integrable on $[0, T]$ and

$$
\left(\mathcal{I H}_{B}\right) \int_{0}^{T}\left\langle W_{t}, \cdot\right\rangle_{U} d W_{t}=\frac{1}{2}\left(\left\|W_{T}\right\|_{U}^{2}+T(\operatorname{tr} Q)\right) .
$$

Proof. We shall consider first the following claims.

Claim 1. Let $D=\left\{\left[v_{i}, \xi_{i}\right]\right\}_{i=1}^{n}$ be a finite collection of disjoint subintervals of $[0, T]$. Then

$$
\mathbb{E}\left[\left|(D) \sum_{i=1}^{n}\left\{\left\|W_{\xi_{i}}-W_{v_{i}}\right\|_{U}^{2}-\left(\xi_{i}-v_{i}\right) \operatorname{tr} Q\right\}\right|^{2}\right]=2 M\left[(D) \sum_{i=1}^{n}\left(\xi_{i}-v_{i}\right)^{2}\right]
$$

where $M=\sum_{j=1}^{\infty} \lambda_{j}^{2}<\infty$.

To verify the first claim,

$$
\begin{gathered}
\mathbb{E}\left[\left|(D) \sum_{i=1}^{n}\left\{\left\|W_{\xi_{i}}-W_{v_{i}}\right\|_{U}^{2}-\left(\xi_{i}-v_{i}\right) \operatorname{tr} Q\right\}\right|^{2}\right] \\
=\sum_{i=1}^{n} \mathbb{E}\left[\left(\left\|W_{\xi_{i}}-W_{v_{i}}\right\|_{U}^{2}-\left(\xi_{i}-v_{i}\right) \operatorname{tr} Q\right)^{2}\right] \\
+2 \sum_{i<p} \mathbb{E}\left[\left(\left\|W_{\xi_{i}}-W_{v_{i}}\right\|_{U}^{2}-\left(\xi_{i}-v_{i}\right) \operatorname{tr} Q\right)\right. \\
\left.\left(\left\|W_{\xi_{p}}-W_{v_{p}}\right\|_{U}^{2}-\left(\xi_{p}-v_{p}\right) \operatorname{tr} Q\right)\right] .
\end{gathered}
$$


Note that

$$
\begin{gathered}
\sum_{i<p} \mathbb{E}\left[\left(\left\|W_{\xi_{i}}-W_{v_{i}}\right\|_{U}^{2}-\left(\xi_{i}-v_{i}\right) \operatorname{tr} Q\right)\left(\left\|W_{\xi_{p}}-W_{v_{p}}\right\|_{U}^{2}-\left(\xi_{p}-v_{p}\right) \operatorname{tr} Q\right)\right] \\
=\sum_{i<p} \mathbb{E}\left[\mathbb { E } \left[\left(\left\|W_{\xi_{i}}-W_{v_{i}}\right\|_{U}^{2}-\left(\xi_{i}-v_{i}\right) \operatorname{tr} Q\right)\right.\right. \\
\left.\left.\quad\left(\left\|W_{\xi_{p}}-W_{v_{p}}\right\|_{U}^{2}-\left(\xi_{p}-v_{p}\right) \operatorname{tr} Q\right) \mid \mathcal{G}_{\xi_{i}}\right]\right] \\
=\sum_{i<p}\left[\left(\xi_{i}-v_{i}\right)\left(\xi_{p}-v_{p}\right)(\operatorname{tr} Q)^{2}-\left(\xi_{i}-v_{i}\right)\left(\xi_{p}-v_{p}\right)(\operatorname{tr} Q)^{2}\right. \\
\left.\quad-\left(\xi_{p}-v_{p}\right)\left(\xi_{i}-v_{i}\right)(\operatorname{tr} Q)^{2}+\left(\xi_{i}-v_{i}\right)\left(\xi_{p}-v_{p}\right)(\operatorname{tr} Q)^{2}\right] \\
=0 .
\end{gathered}
$$

It follows that

$$
\begin{aligned}
\mathbb{E}\left[\left|(D) \sum_{i=1}^{n}\left\{\left\|W_{\xi_{i}}-W_{v_{i}}\right\|_{U}^{2}-\left(\xi_{i}-v_{i}\right) \operatorname{tr} Q\right\}\right|^{2}\right] \\
=\sum_{i=1}^{n} \mathbb{E}\left[\left(\left\|W_{\xi_{i}}-W_{v_{i}}\right\|_{U}^{2}-\left(\xi_{i}-v_{i}\right) \operatorname{tr} Q\right)^{2}\right] .
\end{aligned}
$$

By Theorem 1,

$$
\begin{array}{rl}
\sum_{i=1}^{n} & \mathbb{E}\left[\left(\left\|W_{\xi_{i}}-W_{v_{i}}\right\|_{U}^{2}-\left(\xi_{i}-v_{i}\right) \operatorname{tr} Q\right)^{2}\right] \\
& =\sum_{i=1}^{n} \mathbb{E}\left[\left\|W_{\xi_{i}}-W_{v_{i}}\right\|_{U}^{4}-2\left(\xi_{i}-v_{i}\right)\left\|W_{\xi_{i}}-W_{v_{i}}\right\|_{U}^{2} \operatorname{tr} Q+\left(\xi_{i}-v_{i}\right)^{2}(\operatorname{tr} Q)^{2}\right] \\
& =\sum_{i=1}^{n}\left\{\left(\xi_{i}-v_{i}\right)^{2}\left(2 \sum_{j=1}^{\infty} \lambda_{j}^{2}+(\operatorname{tr} Q)^{2}\right)-2\left(\xi_{i}-v_{i}\right)^{2}(\operatorname{tr} Q)^{2}+\left(\xi_{i}-v_{i}\right)^{2}(\operatorname{tr} Q)^{2}\right\} \\
& =\sum_{i=1}^{n}\left\{\left(\xi_{i}-v_{i}\right)^{2}\left(2 \sum_{j=1}^{\infty} \lambda_{j}^{2}+(\operatorname{tr} Q)^{2}\right)-\left(\xi_{i}-v_{i}\right)^{2}(\operatorname{tr} Q)^{2}\right\} \\
& =\sum_{i=1}^{n}\left\{\left(\xi_{i}-v_{i}\right)^{2}\left(2 \sum_{j=1}^{\infty} \lambda_{j}^{2}+(\operatorname{tr} Q)^{2}-(\operatorname{tr} Q)^{2}\right)\right\} \\
& =\sum_{i=1}^{n}\left\{\left(\xi_{i}-v_{i}\right)^{2}\left(2 \sum_{j=1}^{\infty} \lambda_{j}^{2}\right)\right\} \\
& =\left(2 \sum_{j=1}^{\infty} \lambda_{j}^{2}\right)\left(\sum_{i=1}^{n}\left(\xi_{i}-v_{i}\right)^{2}\right) .
\end{array}
$$


This proves claim 1.

Claim 2. $\mathbb{E}\left[\left\|\left\langle W_{t}, \cdot\right\rangle_{U}\right\|_{L_{2}\left(U_{Q}, \mathbb{R}\right)}^{2}\right]=t M$.

$$
\begin{aligned}
\mathbb{E}\left[\left\|\left\langle W_{t},\right\rangle_{U}\right\|_{L_{2}\left(U_{Q}, \mathbb{R}\right)}^{2}\right] & =\mathbb{E}\left[\sum_{j=1}^{\infty}\left|\left\langle W_{t}, \sqrt{\lambda_{j}} e_{j}\right\rangle_{U}\right|^{2}\right] \\
& =\sum_{j=1}^{\infty} \lambda_{j} \mathbb{E}\left[\left\langle W_{t}-W_{0}, e_{j}\right\rangle_{U}^{2}\right] \\
& =t \sum_{j=1}^{\infty} \lambda_{j}\left\langle\lambda_{j} e_{j}, e_{j}\right\rangle_{U}^{2} \\
& =t \sum_{j=1}^{\infty} \lambda_{j}^{2} \\
& =t M .
\end{aligned}
$$

This proves Claim 2.

Claim 3. $\left\langle W_{t}, \cdot\right\rangle_{U}$ is $\mathcal{I H}_{B}$-integrable to $\frac{1}{2}\left(\left\|W_{T}\right\|_{U}^{2}+T \operatorname{tr} Q\right)$ on $[0, T]$.

Let $\varepsilon>0$ be given. Let $M=\sum_{j=1}^{\infty} \lambda_{j}^{2}$. Choose a constant function $\delta$ on $[0, T]$ defined by $\delta(t)=\frac{\varepsilon}{2 M T}$ and a number $\eta=\frac{\varepsilon}{12 M T}$. Let $D=\{((v, \xi], \xi)\}$ be a backwards $(\delta, \eta)$-fine partial division of $[0, T]$. Let $D^{c}$ be the collection of all subintervals of $[0, T]$ which are not included in $D$. Then

$$
\begin{aligned}
\mathbb{E}\left[\left|(D) \sum\left\langle W_{\xi}, \cdot\right\rangle_{U}\left(W_{\xi}-W_{v}\right)-\frac{1}{2}\left\|W_{T}\right\|_{U}^{2}-\frac{1}{2} T(\operatorname{tr} Q)\right|^{2}\right] \\
=\mathbb{E}\left[\left|(D) \sum\left\langle W_{\xi}, W_{\xi}-W_{v}\right\rangle_{U}-\frac{1}{2}\right|\left|W_{T} \|_{U}^{2}-\frac{1}{2} T(\operatorname{tr} Q)\right|^{2}\right] \\
=\mathbb{E}\left[\mid(D) \sum\left\{\left\langle W_{\xi}, W_{\xi}-W_{v}\right\rangle_{U}-\frac{1}{2}\left(\left\|W_{\xi}\right\|_{U}^{2}-\left\|W_{v}\right\|_{U}^{2}\right)-\frac{1}{2}(\xi-v) \operatorname{tr} Q\right\}\right. \\
\left.\quad+\left.\left(D^{c}\right) \sum\left\{-\frac{1}{2}\left(\left\|W_{\xi}\right\|_{U}^{2}-\left\|W_{v}\right\|_{U}^{2}\right)-\frac{1}{2}(\xi-v) \operatorname{tr} Q\right\}\right|^{2}\right] \\
\leq 2 \mathbb{E}\left[\left|(D) \sum\left\{\left\langle W_{\xi}, W_{\xi}-W_{v}\right\rangle_{U}-\frac{1}{2}\left(\left\|W_{\xi}\right\|_{U}^{2}-\left\|W_{v}\right\|_{U}^{2}\right)-\frac{1}{2}(\xi-v) \operatorname{tr} Q\right\}\right|^{2}\right] \\
\quad+2 \mathbb{E}\left[\left|\left(D^{c}\right) \sum\left\{-\frac{1}{2}\left(\left\|W_{\xi}\right\|_{U}^{2}-\left\|W_{v}\right\|_{U}^{2}\right)-\frac{1}{2}(\xi-v) \operatorname{tr} Q\right\}\right|^{2}\right] \\
=\frac{1}{2} \mathbb{E}\left[\left|(D) \sum\left\{-2\left\langle W_{\xi}, W_{\xi}-W_{v}\right\rangle_{U}+\left\|W_{\xi}\right\|_{U}^{2}-\left\|W_{v}\right\|_{U}^{2}+(\xi-v) \operatorname{tr} Q\right\}\right|^{2}\right]
\end{aligned}
$$




$$
\begin{gathered}
+\frac{1}{2} \mathbb{E}\left[\left|\left(D^{c}\right) \sum\left\{\left\|W_{\xi}\right\|_{U}^{2}-\left\|W_{v}\right\|_{U}^{2}+(\xi-v) \operatorname{tr} Q\right\}\right|^{2}\right] \\
=\frac{1}{2} \mathbb{E}\left[\left|(D) \sum\left\{-\left\|W_{\xi}-W_{v}\right\|_{U}^{2}+(\xi-v) \operatorname{tr} Q\right\}\right|^{2}\right] \\
+\frac{1}{2} \mathbb{E}\left[\mid\left(D^{c}\right) \sum\left\{\left\|W_{\xi}\right\|_{U}^{2}-\left\|W_{v}\right\|_{U}^{2}-2\left\langle W_{\xi}, W_{\xi}-W_{v}\right\rangle_{U}\right.\right. \\
\left.\left.+2\left\langle W_{\xi}, W_{\xi}-W_{v}\right\rangle_{U}+(\xi-v) \operatorname{tr} Q\right\}\left.\right|^{2}\right] \\
\leq \frac{1}{2} \mathbb{E}\left[\left|(D) \sum\left\{|| W_{\xi}-W_{v} \|_{U}^{2}-(\xi-v) \operatorname{tr} Q\right\}\right|^{2}\right] \\
+\mathbb{E}\left[\left|\left(D^{c}\right) \sum\left\{|| W_{\xi}-W_{v} \|_{U}^{2}-(\xi-v) \operatorname{tr} Q\right\}\right|^{2}\right] \\
+4 \mathbb{E}\left[\left|\left(D^{c}\right) \sum\left\langle W_{\xi}, W_{\xi}-W_{v}\right\rangle_{U}\right|^{2}\right] .
\end{gathered}
$$

By Claim 1, Claim 2, and Lemma 2, we have

$$
\begin{aligned}
\mathbb{E}[\mid(D) & \left.\sum\left\langle W_{\xi},\right\rangle_{U}\left(W_{\xi}-W_{v}\right)-\frac{1}{2}\left\|W_{T}\right\|_{U}^{2}-\left.\frac{1}{2} T(\operatorname{tr} Q)\right|^{2}\right] \\
\leq & M\left[(D) \sum(\xi-v)^{2}\right]+2 M\left[\left(D^{c}\right) \sum(\xi-v)^{2}\right]+4 \cdot\left(D^{c}\right) \sum(\xi-v) \xi M \\
& <M T \delta+2 M T \eta+4 M T \eta \\
& =M T\left(\frac{\varepsilon}{2 M T}\right)+6 M T\left(\frac{\varepsilon}{12 M T}\right) \\
& =\varepsilon .
\end{aligned}
$$

Thus, $\left\langle W_{t}, \cdot\right\rangle_{U}$ is $\mathcal{I H}_{B}$-integrable on $[0, T]$ and

$$
\left(\mathcal{I H}_{B}\right) \int_{0}^{T}\left\langle W_{t}, \cdot\right\rangle_{U} d W_{t}=\frac{1}{2}\left(\left\|W_{T}\right\|_{U}^{2}+T \operatorname{tr} Q\right) .
$$

The following statements show that the backwards Itô-Henstock integral possesses the standard properties of an integral. Refer to [12] for analogous proofs.

(1) The backwards Itô-Henstock integral is uniquely determined, in the sense that if $A_{1}$ and $A_{2}$ are two backwards Itô-Henstock integrals of $f$ in Definition 1 , then $\left\|A_{1}-A_{2}\right\|_{L^{2}(\Omega, V)}=0$.

(2) Let $\alpha \in \mathbb{R}$. If $f$ and $g$ are $\mathcal{I H}_{B}$-integrable on $[0, T]$, then

(i) $f+g$ is $\mathcal{I H}_{B}$-integrable on $[0, T]$, and

$$
\left(\mathcal{I H}_{B}\right) \int_{0}^{T}(f+g) d W=\left(\mathcal{I H}_{B}\right) \int_{0}^{T} f d W+\left(\mathcal{I H}_{B}\right) \int_{0}^{T} g d W
$$


(ii) $\alpha f$ is $\mathcal{I H}_{B}$-integrable on $[0, T]$, and

$$
\left(\mathcal{I H}_{B}\right) \int_{0}^{T}(\alpha f) d W=\alpha \cdot\left(\mathcal{I H}_{B}\right) \int_{0}^{T} f d W .
$$

(3) If $f:[0, T] \times \Omega \rightarrow L_{2}\left(U_{Q}, V\right)$ is $\mathcal{I H}_{B}$-integrable on $[0, c]$ and $[c, T]$ where $c \in(0, T)$, then $f$ is $\mathcal{I H}_{B}$-integrable on $[0, T]$ and

$$
\left(\mathcal{I H}_{B}\right) \int_{0}^{T} f d W=\left(\mathcal{I H}_{B}\right) \int_{0}^{c} f d W+\left(\mathcal{I H}_{B}\right) \int_{c}^{T} f d W .
$$

(4) If $f:[0, T] \times \Omega \rightarrow L_{2}\left(U_{Q}, V\right)$ is $\mathcal{I H}_{B}$-integrable on $[0, T]$, then $f$ is also $\mathcal{I H}_{B}$-integrable on every subinteval $[c, d]$ of $[0, T]$.

(5) A process $f:[0, T] \times \Omega \rightarrow L_{2}\left(U_{Q}, V\right)$ is $\mathcal{I H}_{B}$-integrable on $[0, T]$ if and only if there exist $A \in L^{2}(\Omega, V)$, a decreasing sequence $\left\{\delta_{n}\right\}$ of positive functions defined on $(0, T]$, and a decreasing sequence of positive numbers $\left\{\eta_{n}\right\}$ such that for any backwards $\left(\delta_{n}, \eta_{n}\right)$-fine partial division $D_{n}$ of $[0, T]$, we have

$$
\lim _{n \rightarrow \infty} \mathbb{E}\left[\left\|S\left(f, D_{n}, \delta_{n}, \eta_{n}\right)-A\right\|_{V}^{2}\right]=0 .
$$

In this case,

$$
A=\left(\mathcal{I H}_{B}\right) \int_{0}^{T} f_{t} d W_{t}
$$

(6) (Cauchy criterion). A process $f:[0, T] \times \Omega \rightarrow L_{2}\left(U_{Q}, V\right)$ is $\mathcal{I H}_{B}$-integrable on $[0, T]$ if and only if for every $\varepsilon>0$, there exist a positive function $\delta$ on $(0, T]$ and a positive number $\eta$ such that for any two backwards $(\delta, \eta)$-fine partial divisions $D$ and $D^{\prime}$ of $[0, T]$, we have

$$
\mathbb{E}\left[\left\|S(f, D, \delta, \eta)-S\left(f, D^{\prime}, \delta, \eta\right)\right\|_{V}^{2}\right]<\varepsilon
$$

(7) (Weak Version of Saks-Henstock Lemma). Let $f$ be $\mathcal{I H}_{B}$-integrable on $[0, T]$ and $F(u, v):=\left(\mathcal{I H}_{B}\right) \int_{u}^{v} f_{t} d W_{t}$ for any $(u, v] \subseteq[0, T]$. Then for every $\varepsilon>0$, there exist a positive function $\delta$ on $(0, T]$ and a positive number $\eta$ such that for any backwards $(\delta, \eta)$-fine partial division $D$ of $[0, T]$, we have

$$
\mathbb{E}\left[\left\|(D) \sum\left\{f_{\xi}\left(W_{\xi}-W_{v}\right)-F(v, \xi)\right\}\right\|_{V}^{2}\right]<\varepsilon .
$$




\section{Itô Isometry and $A C^{2}[0, T]$-property}

This section presents the Itô isometry and the equivalent definition of backwards ItôHenstock using the notion of $A C^{2}[0, T]$-property. Before we proceed with the Itô isometry, we need to define the backwards Henstock integral which is equivalent to the Lebesgue integral (see [2]).

Definition 2. A real-valued function $f$ defined on $[0, T]$ is said to be Lebesgue integrable to $A \in \mathbb{R}$ if given $\varepsilon>0$, there exists a positive function $\delta$ on $(0, T]$ and a real constant $\eta>0$ such that

$$
\left|(D) \sum f(\xi)(\xi-v)-A\right|<\varepsilon
$$

whenever $D$ is a backwards $\delta$-fine partial division of $[0, T]$ with $(D) \sum(\xi-v)>T-\eta$. In this case, $A$ is called the Lebesgue integral of $f$ which will be denoted by $(\mathcal{L}) \int_{0}^{T} f(t) d t$.

Note that the backwards $\delta$-fine partial division $D$ of $[0, T]$ in Definition 2 is also a backwards $(\delta, \eta)$-fine partial division of $[0, T]$.

Theorem 2. The function $f:[0, T] \rightarrow \mathbb{R}$ is Lebesgue integrable to $A \in \mathbb{R}$ if and only if there exists a decreasing sequence of positive functions $\left\{\delta_{n}(\xi)\right\}$ on $(0, T]$ and a decreasing sequence of positive constants $\left\{\eta_{n}\right\}$ such that

$$
\lim _{n \rightarrow \infty}\left|\left(D_{n}\right) \sum f\left(\xi^{(n)}\right)\left(\xi^{(n)}-v^{(n)}\right)-A\right|=0,
$$

where $D_{n}$ is any backwards $\left(\delta_{n}, \eta_{n}\right)$-fine partial division of $[0, T]$.

Proof. Suppose that $f:[0, T] \rightarrow \mathbb{R}$ is Lebesgue integrable to $A \in \mathbb{R}$. Then, by Definition 2, for every $\varepsilon=\frac{1}{n}, n=1,2,3, \ldots$, there exists a positive function $\delta_{n}$ on $(0, T]$ and a positive number $\eta$ such that for any backwards $\delta_{n}$-fine partial division $D_{n}=\left\{\left(\left(v^{(n)}, \xi^{(n)}\right], \xi^{(n)}\right)\right\}$ of $[0, T]$ with $\left(D_{n}\right) \sum\left(\xi^{(n)}-v^{(n)}\right)>T-\eta_{n}$ we have

$$
\left|\left(D_{n}\right) \sum f\left(\xi^{(n)}\right)\left(\xi^{(n)}-v^{(n)}\right)-A\right| \leq \frac{1}{n} .
$$

Hence,

$$
\lim _{n \rightarrow \infty}\left|\left(D_{n}\right) \sum f\left(\xi^{(n)}\right)\left(\xi^{(n)}-v^{(n)}\right)-A\right|=0,
$$

for any backwards $\left(\delta_{n}, \eta_{n}\right)$-fine partial division $D_{n}$ of $[0, T]$.

Conversely, let us assume that there exists $A \in \mathbb{R}$ and a decreasing sequence $\left\{\delta_{n}(\xi)\right\}$ of positive functions on $(0, T]$ and a decreasing sequence of positive numbers $\left\{\eta_{n}\right\}$ such that

$$
\lim _{n \rightarrow \infty}\left|\left(D_{n}\right) \sum f\left(\xi^{(n)}\right)\left(\xi^{(n)}-v^{(n)}\right)-A\right|=0
$$

Suppose that $f$ is not Lebesgue integrable to $A$ on $[0, T]$. Then there exists $\varepsilon>0$ such that for every positive function $\delta$ on $(0, T]$ and every positive number $\eta$ there exists a 
backwards $\delta$-fine partial division $D=\{((v, \xi], \xi)\}$ of $[0, T]$ with $(D) \sum(\xi-v)>T-\eta$ such that

$$
\left|(D) \sum f(\xi)(\xi-v)-A\right| \geq \varepsilon \text {. }
$$

Hence, for each $\delta_{n}$ and $\eta_{n}$, there exists a $\delta_{n}$-fine partial division $D_{n}$ of $[0, T]$ with

$$
\left(D_{n}\right) \sum(\xi-v)>T-\eta_{n} \text { such that }\left|\left(D_{n}\right) \sum f(\xi)(\xi-v)-A\right| \geq \varepsilon,
$$

leading to a contradiction.

We now state and prove the Itô isometry.

Theorem 3 (Itô Isometry). Let $f$ be $\mathcal{I H}_{B}$-integrable on $[0, T]$. Then $\mathbb{E}\left[\left\|f_{t}\right\|_{L_{2}\left(U_{Q}, V\right)}^{2}\right]$ is Lebesgue integrable on $[0, T]$ and

$$
\mathbb{E}\left[\left\|\left(\mathcal{I H}_{B}\right) \int_{0}^{T} f_{t} d W_{t}\right\|_{V}^{2}\right]=(\mathcal{L}) \int_{0}^{T} \mathbb{E}\left[\left\|f_{t}\right\|_{L_{2}\left(U_{Q}, V\right)}^{2}\right] d t<\infty .
$$

Proof. From property (5) section 3, there exists a decreasing sequence $\left\{\delta_{n}(\xi)\right\}$ of positive functions defined on $(0, T]$, and a decreasing sequence of positive numbers $\left\{\eta_{n}\right\}$ such that for any backwards $\left(\delta_{n}, \eta_{n}\right)$-fine partial division $\left.D_{n}=\left\{\left(\left(v_{i}^{(n)}, \xi_{i}^{(n)}\right)\right], \xi_{i}^{(n)}\right)\right\}_{i=1}^{p(n)}$ of $[0, T]$, we have

$$
\lim _{n \rightarrow \infty} \mathbb{E}\left[\left\|S\left(f, D_{n}, \delta_{n}, \eta_{n}\right)-\left(\mathcal{I H}_{B}\right) \int_{0}^{T} f_{t} d W_{t}\right\|_{V}^{2}\right]=0 .
$$

This means that

$$
\lim _{n \rightarrow \infty} S\left(f, D_{n}, \delta_{n}, \eta_{n}\right)=\left(\mathcal{I H}_{B}\right) \int_{0}^{T} f_{t} d W_{t} \quad \text { in } L^{2}(\Omega, V) .
$$

Let $\varepsilon>0$ be given. Then there exists $N \in \mathbb{N}$ such that for all $n \geq N$,

$$
\left\|S\left(f, D_{n}, \delta_{n}, \eta_{n}\right)-\left(\mathcal{I H}_{B}\right) \int_{0}^{T} f_{t} d W_{t}\right\|_{L^{2}(\Omega, V)}<\varepsilon
$$

Note that

$$
\begin{gathered}
\left|\left\|S\left(f, D_{n}, \delta_{n}, \eta_{n}\right)\right\|_{L^{2}(\Omega, V)}-\left\|\left(\mathcal{I H}_{B}\right) \int_{0}^{T} f_{t} d W_{t}\right\|_{L^{2}(\Omega, V)}\right| \\
\leq\left\|S\left(f, D_{n}, \delta_{n}, \eta_{n}\right)-\left(\mathcal{I H}_{B}\right) \int_{0}^{T} f_{t} d W_{t}\right\|_{L^{2}(\Omega, V)}
\end{gathered} .
$$

This implies that

$$
\lim _{n \rightarrow \infty}\left\|S\left(f, D_{n}, \delta_{n}, \eta_{n}\right)\right\|_{L^{2}(\Omega, V)}=\left\|\left(\mathcal{I H}_{B}\right) \int_{0}^{T} f_{t} d W_{t}\right\|_{L^{2}(\Omega, V)}
$$




$$
\lim _{n \rightarrow \infty} \sqrt{\mathbb{E}\left[\left\|S\left(f, D_{n}, \delta_{n}, \eta_{n}\right)\right\|_{V}^{2}\right]}=\sqrt{\mathbb{E}\left[\left\|\left(\mathcal{I H}_{B}\right) \int_{0}^{T} f_{t} d W_{t}\right\|_{V}^{2}\right]} .
$$

Using Lemma 2, we have

$$
\begin{aligned}
\sqrt{\mathbb{E}\left[\left\|\left(\mathcal{I H}_{B}\right) \int_{0}^{T} f_{t} d W_{t}\right\|_{V}^{2}\right]} & =\lim _{n \rightarrow \infty} \sqrt{\mathbb{E}\left[\left\|S\left(f, D_{n}, \delta_{n}, \eta_{n}\right)\right\|_{V}^{2}\right]} \\
& =\lim _{n \rightarrow \infty} \sqrt{\mathbb{E}\left[\left\|\sum_{i=1}^{p(n)} f_{\xi_{i}^{(n)}}\left(W_{\xi_{i}^{(n)}}-W_{v_{i}^{(n)}}\right)\right\|_{V}^{2}\right]} \\
& =\lim _{n \rightarrow \infty} \sqrt{\sum_{i=1}^{p(n)}\left(\xi_{i}^{(n)}-v_{i}^{(n)}\right) \mathbb{E}\left[\left\|f_{\xi_{i}^{(n)}}\right\|_{L_{2}\left(U_{Q}, V\right)}^{2}\right]} .
\end{aligned}
$$

This implies that

$$
\lim _{n \rightarrow \infty} \sum_{i=1}^{p(n)}\left(\xi_{i}^{(n)}-v_{i}^{(n)}\right) \mathbb{E}\left[\left\|f_{\xi_{i}^{(n)}}\right\|_{L_{2}\left(U_{Q}, V\right)}^{2}\right]=\mathbb{E}\left[\left\|\left(\mathcal{I H}_{B}\right) \int_{0}^{T} f_{t} d W_{t}\right\|_{V}^{2}\right] .
$$

Since the above equality holds for any backwards $\left(\delta_{n}, \eta_{n}\right)$-fine partial division of $[0, T]$, by Theorem 2, $\mathbb{E}\left[\left\|f_{\xi_{i}^{(n)}}\right\|_{L_{2}\left(U_{Q}, V\right)}^{2}\right]$ is Lebesgue integrable on $[0, T]$ and

$$
\mathbb{E}\left[\left\|\left(\mathcal{I H}_{B}\right) \int_{0}^{T} f_{t} d W_{t}\right\|_{V}^{2}\right]=(\mathcal{L}) \int_{0}^{T} \mathbb{E}\left[\left\|f_{t}\right\|_{L_{2}\left(U_{Q}, V\right)}^{2}\right] d t<\infty .
$$

Throughout the following, denote by $\mathcal{J}$ the family of all left-open subintervals $(v, \xi]$ of $[0, T]$. In the following, when no confusion arises, we may refer to $F((u, v], \cdot)$ or $F((u, v], \omega)$ as simply $F(u, v)$.

Definition 3. A function $F: \mathcal{J} \times \Omega \rightarrow V$ is said to be $A C^{2}[0, T]$ if for every $\varepsilon>0$, there exists $\eta>0$ such that for any finite collection $D=\{(v, \xi]\}$ of disjoint subintervals $(v, \xi] \in J$ with

$$
(D) \sum(\xi-v)<\eta
$$

we have

$$
\int_{\Omega}\left\|(D) \sum F((v, \xi), \omega)\right\|_{V}^{2} d \mathbb{P}(\omega):=\mathbb{E}\left[\left\|(D) \sum F(v, \xi)\right\|_{V}^{2}\right]<\varepsilon
$$


Lemma 3. Let $f$ be $\mathcal{I H}_{B}$-integrable on $[0, T]$. Then for every $\varepsilon>0$, there exist a positive function $\delta$ on $(0, T]$ and a positive number $\eta$ such that

$$
\mathbb{E}\left[\left\|(D) \sum f_{\xi}\left(W_{\xi}-W_{v}\right)\right\|_{V}^{2}\right]<\varepsilon
$$

for any backwards $\delta$-fine partial division $D=\{((v, \xi], \xi)\}$ of $[0, T]$ with

$$
\text { (D) } \sum|\xi-v| \leq \eta \text {. }
$$

Proof. Let $\varepsilon>0$ be given. Then there exist a positive function $\delta$ on $(0, T]$ and a positive number $\eta$ such that for any backwards $(\delta, \eta)$-fine partial division $P$ of $[0, T]$, we have

$$
\mathbb{E}\left[\left\|S(f, P, \delta, \eta)-\left(\mathcal{I H}_{B}\right) \int_{0}^{T} f_{t} d W_{t}\right\|_{V}^{2}\right]<\frac{\varepsilon}{4} .
$$

Let $D=\{((v, \xi], \xi)$ be a backwards $\delta$-fine partial division of $[0, T]$ with

$$
\text { (D) } \sum|\xi-v| \leq \eta
$$

Construct a backwards $(\delta, \eta)$-fine partial division $D_{1}$ of $[0, T]$ such that $D$ and $D_{1}$ are disjoint and $D \cup D_{1}$ is a backwards $(\delta, \eta)$-fine partial division of $[0, T]$. By assumption,

$$
\mathbb{E}\left[\left\|\left(D \cup D_{1}\right) \sum f_{\xi}\left(W_{\xi}-W_{v}\right)-\left(\mathcal{I} \mathcal{H}_{B}\right) \int_{0}^{T} f_{t} d W_{t}\right\|_{V}^{2}\right]<\frac{\varepsilon}{4} .
$$

Hence,

$$
\begin{aligned}
\mathbb{E}\left[\left\|(D) \sum f_{\xi}\left(W_{\xi}-W_{v}\right)\right\|_{V}^{2}\right] \\
=\mathbb{E}\left[\|\left(D \cup D_{1}\right) \sum f_{\xi}\left(W_{\xi}-W_{v}\right)-\left(\mathcal{I} \mathcal{H}_{B}\right) \int_{0}^{T} f_{t} d W_{t}\right. \\
\left.\quad+\left(\mathcal{I} \mathcal{H}_{B}\right) \int_{0}^{T} f_{t} d W_{t}-\left(D_{1}\right) \sum f_{\xi}\left(W_{\xi}-W_{v}\right) \|_{V}^{2}\right] \\
\leq 2 \mathbb{E}\left[\left\|\left(D \cup D_{1}\right) \sum f_{\xi}\left(W_{\xi}-W_{v}\right)-\left(\mathcal{I} \mathcal{H}_{B}\right) \int_{0}^{T} f_{t} d W_{t}\right\|_{V}^{2}\right] \\
\quad+2 \mathbb{E}\left[\left\|\left(\mathcal{I H}_{B}\right) \int_{0}^{T} f_{t} d W_{t}-\left(D_{1}\right) \sum f_{\xi}\left(W_{\xi}-W_{v}\right)\right\|_{V}^{2}\right] \\
<2\left(\frac{\varepsilon}{4}\right)+2\left(\frac{\varepsilon}{4}\right) \\
=\varepsilon .
\end{aligned}
$$

This proves the lemma. 
Theorem 4. Let $f$ be $\mathcal{I H}_{B}$-integrable on $[0, T]$ and define

$$
F(v, \xi):=\left(\mathcal{I H}_{B}\right) \int_{v}^{\xi} f_{t} d W_{t}
$$

for all $(v, \xi] \in \mathcal{J}$. Then $F$ is $A C^{2}[0, T]$.

Proof. Let $\varepsilon>0$ be given. By Lemma 3, there exist a positive function $\delta$ on $(0, T]$ and a positive number $\eta$ such that

$$
\mathbb{E}\left[\left\|(D) \sum f_{\xi}\left(W_{\xi}-W_{v}\right)\right\|_{V}^{2}\right]<\frac{\varepsilon}{4}
$$

for any backwards $\delta$-fine partial division $D=\{((v, \xi], \xi)\}$ of $[0, T]$ with

$$
\text { (D) } \sum|\xi-v| \leq \eta \text {. }
$$

Let $\left\{\left(a_{j}, b_{j}\right]\right\}_{j=1}^{m}$ be a finite collection of disjoint subintervals $\left(a_{j}, b_{j}\right] \in \mathcal{J}$ with $\sum_{j=1}^{m} \mid b_{j}-$ $a_{j} \mid \leq \eta$. By property (4) section $3, f$ is also $\mathcal{I H}_{B}$-integrable on $\left[a_{j}, b_{j}\right]$ for all $j$. This means that for all $j$, there exist positive function $\delta_{j}$ on $\left(a_{j}, b_{j}\right]$ and a positive number $\eta_{j}$ such that for any backwards $\left(\delta_{j}, \eta_{j}\right)$-fine partial division $D_{j}$ of $\left[a_{j}, b_{j}\right]$, we have

$$
\mathbb{E}\left[\left\|S\left(f, D_{j}, \delta_{j}, \eta_{j}\right)-F\left(a_{j}, b_{j}\right)\right\|_{V}^{2}\right]<\frac{\varepsilon}{4 \cdot 2^{2 j}} .
$$

We can choose $\left\{\delta_{j}\right\}_{j=1}^{m}$ and $\left\{\eta_{j}\right\}_{j=1}^{m}$ such that $\delta_{j}(\xi) \leq \delta(\xi)$ for all $j$ and $\sum_{j=1}^{m} \eta_{j} \leq \eta$. Let $P=D_{1} \cup D_{2} \cup \cdots \cup D_{m}$, which is a backwards $\delta$-fine partial division of $[0, T]$ with

$$
\text { (P) } \sum|\xi-v| \leq \sum_{j=1}^{m}\left|b_{j}-a_{j}\right| \leq \eta .
$$

This implies that

$$
\mathbb{E}\left[\left\|(P) \sum f_{\xi}\left(W_{\xi}-W_{v}\right)\right\|_{V}^{2}\right]<\frac{\varepsilon}{4}
$$

Hence,

$$
\begin{array}{r}
\mathbb{E}\left[\left\|\sum_{j=1}^{m} F\left(a_{j}, b_{j}\right)\right\|_{V}^{2}\right] \\
\leq 2 \mathbb{E}\left[\left\|\sum_{j=1}^{m}\left\{F\left(a_{j}, b_{j}\right)-S\left(f, D_{j}, \delta_{j}, \eta_{j}\right)\right\}\right\|_{V}^{2}\right] \\
+2 \mathbb{E}\left[\left\|\sum_{j=1}^{m} S\left(f, D_{j}, \delta_{j}, \eta_{j}\right)\right\|_{V}^{2}\right]
\end{array}
$$




$$
\begin{aligned}
& =2\left\|\sum_{j=1}^{m}\left\{F\left(a_{j}, b_{j}\right)-S\left(f, D_{j}, \delta_{j}, \eta_{j}\right)\right\}\right\|_{L^{2}(\Omega, V)}^{2} \\
& \quad+2 \mathbb{E}\left[\left\|(P) \sum f_{\xi}\left(W_{\xi}-W_{v}\right)\right\|_{V}^{2}\right] \\
& =2\left(\sum_{j=1}^{m} \sqrt{\mathbb{E}\left[\left\|F\left(a_{j}, b_{j}\right)-S\left(f, D_{j}, \delta_{j}, \eta_{j}\right)\right\|_{V}^{2}\right]}\right)^{2} \\
& \quad+2 \mathbb{E}\left[\left\|(P) \sum f_{\xi}\left(W_{\xi}-W_{v}\right)\right\|_{V}^{2}\right] \\
& <2\left(\sum_{j=1}^{m} \frac{\sqrt{\varepsilon}}{2 \cdot 2^{j}}\right)^{2}+2\left(\frac{\varepsilon}{4}\right) \\
& <\varepsilon .
\end{aligned}
$$

Thus, $F$ is $A C^{2}[0, T]$.

The following result provides an equivalent definition of an $\mathcal{I H}_{B}$-integrable process using $A C^{2}$ property.

Theorem 5. Let $f:[0, T] \times \Omega \rightarrow L_{2}\left(U_{Q}, V\right)$ be a backwards process. Then $f$ is $\mathcal{I H}_{B^{-}}$ integrable on $[0, T]$ if and only if there exists an $A C^{2}[0, T]$ function $F$ such that for every $\varepsilon>0$, there exist a positive function $\delta$ on $(0, T]$ such that whenever $D=\{((v, \xi], \xi)\}$ is a backwards $\delta$-fine partial division of $[0, T]$, we have

$$
\mathbb{E}\left[\left\|(D) \sum\left\{f_{\xi}\left(W_{\xi}-W_{v}\right)-F(v, \xi)\right\}\right\|_{V}^{2}\right]<\varepsilon .
$$

Proof. Suppose that $f$ is $\mathcal{I H}_{B}$-integrable on $[0, T]$. By Theorem 4 and property (7) section 3 , the result follows.

For the converse, let $\varepsilon>0$ be given. Since $F$ is $A C^{2}[0, T]$, choose $\eta>0$ such that whenever $\left\{\left(v_{j}, \xi_{j}\right]\right\}_{j=1}^{m}$ is a finite collection of subintervals $\left(v_{j}, \xi_{j}\right] \in \mathcal{J}$ with

$$
\sum_{j=1}^{m}\left|\xi_{j}-v_{j}\right| \leq \eta
$$

we have

$$
\mathbb{E}\left[\left\|\sum_{j=1}^{m} F\left(v_{j}, \xi_{j}\right)\right\|_{V}^{2}\right]<\frac{\varepsilon}{4} .
$$

Let $D=\{((v, \xi], \xi)\}$ be a backwards $(\delta, \eta)$-fine partial division of $[0, T]$ and let $D^{c}$ be the collection of all subintervals of $[0, T]$ which are not included in the set $D$. Since $F$ is $A C^{2}[0, T]$,

$$
\mathbb{E}\left[\left\|\left(D^{c}\right) \sum F(v, \xi)\right\|_{V}^{2}\right]<\frac{\varepsilon}{4} .
$$


Hence,

$$
\begin{aligned}
\mathbb{E}\left[\left\|(D) \sum f_{\xi}\left(W_{\xi}-W_{v}\right)-F(0, T)\right\|_{V}^{2}\right] \\
=\mathbb{E}\left[\left\|(D) \sum\left\{f_{\xi}\left(W_{\xi}-W_{v}\right)-F(v, \xi)\right\}-\left(D^{c}\right) \sum F(v, \xi)\right\|_{V}^{2}\right] \\
\leq 2 \mathbb{E}\left[\left\|(D) \sum\left\{f_{\xi}\left(W_{\xi}-W_{v}\right)-F(v, \xi)\right\}\right\|_{V}^{2}\right] \\
\quad+2 \mathbb{E}\left[\left\|\left(D^{c}\right) \sum F(v, \xi)\right\|_{V}^{2}\right] \\
<2\left(\frac{\varepsilon}{4}\right)+2\left(\frac{\varepsilon}{4}\right) \\
=\varepsilon .
\end{aligned}
$$

Thus, $f$ is $\mathcal{I H}_{B}$-integrable on $[0, T]$.

\section{Conclusion and Recommendation}

In this paper, we formulate the Itô isometry for the backwards Itô-Henstock integral of an operator-valued stochastic process with respect to a Hilbert space-valued $Q$-Wiener process and provide an equivalent definition for this integral using the concept $A C^{2}[0, T]$ property, a version of absolute continuity. A worthwhile direction for further investigation is to formulate a version of Itô's formula for this type of integral.

\section{Acknowledgement}

The authors would like to acknowledge the financial support from the Department of Science and Technology-Accelerated Science and Technology Human Resource Development Program (DOST-ASTHRDP) and to thank the unknown referee for the helpful comments for the improvement of this paper.

\section{References}

[1] D. Applebaum. Lévy Processes and Stochastic Calculus. Cambridge University Press, New York, 2004.

[2] J. Arcede and E. Cabral. Backwards Henstock integral. MATIMYÁS MATEMATIKA, Journal of the Mathematical Society of the Philippines, 32:1-12, 2009.

[3] J. Arcede and E. Cabral. An equivalent definition for the backwards Itô integral. Thai Journal of Mathematics, 9:619-630, 2011. 
[4] J. Arcede and E. Cabral. Fundamental Theorem of Calculus for the backwards Itô integral. MATIMYÁS MATEMATIKA, Journal of the Mathematical Society of the Philippines, 34:1-9, 2011.

[5] J. Arcede and E. Cabral. On integration-by-parts and Itô formula for backwards Itô integral. The MINDANAWAN Journal of Mathematics, 3:112-132, 2012.

[6] L. Gawarecki and V. Mandrekar. Stochastic Differential Equations in Infinite Dimensions with Applications to Stochastic Partial Differential Equations. Springer, Berlin, 2011.

[7] R. A. Gordon. The Integrals of Lebesgue, Denjoy, Perron and Henstock. American Mathematical Society, 1994.

[8] R. Henstock. Lectures on the Theory of Integration. World Scientific, Singapore, 1988.

[9] J. Kurzweil. Henstock-Kurzweil Integration: Its Relation to Topological Vector Spaces. World Scientific, Singapore, 2000.

[10] M. Labendia and J. Benitez. Convergence theorems for the Itô-Henstock integrable operator-valued stochastic process. Malaysian Journal of Mathematical Sciences, to appear.

[11] M. Labendia E. De Lara-Tuprio and T. R. Teng. Itô-Henstock integral and Itô's formula for the operator-valued stochastic process. Mathematica Bohemica, 143:135$160,2018$.

[12] P. Y. Lee. Lanzhou Lectures on Henstock Integration. World Scientific, Singapore, 1989.

[13] P. Y. Lee and R. Výborný. The Integral: An Easy Approach after Kurzweil and Henstock. Cambridge University Press, Cambridge, 2000.

[14] T. Y. Lee. Henstock-Kurzweil Integration on Euclidean Spaces. World Scientific, Singapore, 2011.

[15] E. J. McShane. Stochastic integrals and stochastic functional equations. SIAM J. Appl. Math., 17:287-306, 1969.

[16] B. Øksendal. Stochastic Differential Equations (5th edition). Springer-Verlag, New York, 1998.

[17] Z. R. Pop-Stojanovic. On Mcshane's belated stochastic integral. SIAM J. Appl. Math., 22:87-92, 1972.

[18] G. Da Prato and J. Zabczyk. Stochastic Equations in Infinite Dimensions. Cambridge University Press, Cambridge, 1992. 
[19] C. Prévôt and M. Röckner. A Concise Course on Stochastic Partial Differential Equations. 2007.

[20] M. Reed and B. Simon. Methods of Modern Mathematical Physics I: Functional Analysis. 1980.

[21] T. L. Toh and T. S. Chew. The Riemann approach to stochastic integration using non-uniform meshes. J. Math. Anal. Appl., 280:133-147, 2003.

[22] T. L. Toh and T. S. Chew. On the Henstock-Fubini theorem for multiple stochastic integrals. Real Anal. Exchange, 30:295-310, 2004-2005.

[23] T. S. Chew T. L. Toh and J. Y. Tay. The non-uniform Riemann approach to Itô's integral. Real Anal. Exchange, 27:495-514, 2002-2003. 